Proceedings of the 8th Workshop on Quantum Chaos and Localisation Phenomena, May 19-21, 2017, Warsaw, Poland

\title{
Surgery of Graphs: $M$-Function and Spectral Gap
}

\author{
P. KURASOV* \\ Dept. of Mathematics, Stockholm Univ., 106 91, Stockholm, Sweden
}

\begin{abstract}
We discuss behaviour of the spectral gap for quantum graphs when two metric graphs are glued together. It appears that precise answer to this question can be given using a natural generalisation of the Titchmarsh-Weyl $M$-functions.
\end{abstract}

DOI: 10.12693/APhysPolA.132.1666

PACS/topics: quantum graphs, spectral theory

\section{Introduction}

Spectral properties of quantum graphs are closely related to topological and geometric properties of the underlying metric graph $[1,2]$. Therefore it appears important to study behaviour of the spectrum under the change of the metric graph. Different transformations of the underlying metric graph have been considered [311]. Our goal today is to understand what happens to the spectrum if the graph is cut into two or more pieces or if two or more graphs are glued into one. We call such changes in the metric graph structure by surgery. This is a hot topic these days, but the most relevant source to our opinion is the recent paper [12], where approach based on the generalisation of the Titchmarsh-Weyl $M$-functions is developed. That paper uses sophisticated theory of such matrix-valued functions, but it appears that many properties of these functions follow directly from their representations in terms of the Laplacian eigenfunctions. The goal of the current paper is to make an elementary introduction into this theory drastically simplifying [20] and to provide illustrative examples.

\section{Quantum graphs - brief introduction}

\subsection{Definition of the operator}

Under quantum graphs we understand ordinary differential equations on metric graphs coupled via certain vertex conditions [13-15]. In the current paper we understand the metric graph $\Gamma$ as a union of compact intervals $\left[x_{2 n-1}, x_{2 n}\right], n=1,2, \ldots, N$ with end-points belonging to the same vertex identified. Each vertex $V_{m}, m=1,2, \ldots, M$, can be understood as an equivalence class of end-points $V_{m} \subset \boldsymbol{V}=\left\{x_{j}\right\}_{j=1}^{2 N}$. Of course we assume that $V_{m} \cap V_{m^{\prime}}=\emptyset$, provided $m \neq m^{\prime}$ and $\cup_{m=1}^{M} V_{m}=\boldsymbol{V}$.

We restrict our studies here to the Laplace differential operator

$$
L=-\frac{\mathrm{d}^{2}}{\mathrm{~d} x^{2}}
$$

*e-mail: kurasov@math.su.se defined as a self-adjoint operator in the Hilbert space

$$
L_{2}(\Gamma)=\oplus_{n=1}^{N} L_{2}\left[x_{2 n-1}, x_{2 n}\right] .
$$

The domain of this operator consists of all functions having square integrable second derivative (in the generalised sense) and satisfying standard vertex conditions at every vertex $V_{m}$. Standard vertex conditions mean that the function is continuous and its first derivatives satisfy the following balance equation:

$$
\sum_{x_{j} \in V_{m}} \partial u\left(x_{j}\right)=0
$$

where $\partial u\left(x_{j}\right)$ denotes the normal derivatives at $x_{j}$ - the limiting values of the first derivative taken in the direction inside the edge. The limit is taken from inside the edge. Standard conditions at vertices of degree one is nothing else than the usual Neumann conditions. In addition we are going to use the Dirichlet conditions, but only at vertices of degree one. (The Dirichlet conditions imposed at any vertex of larger degree imply that the vertex is decomposed into a set of independent vertices of degree one with the Dirichlet vertex conditions.) The standard Laplacian on a metric graph $\Gamma$ is uniquely determined by the graph, therefore we are going to call its spectrum by spectrum of $\Gamma$.

\subsection{Elementary spectral properties}

The spectrum of a quantum graph is discrete and tends to $\infty$ satisfying Weyl's asymptotic law

$$
\lambda_{n} \sim\left(\frac{\pi}{\mathcal{L}}\right)^{2} n^{2}
$$

where $\mathcal{L}$ is the total length of the graph

$$
\mathcal{L}=\sum_{n=1}^{N}\left(x_{2 n}-x_{2 n-1}\right) .
$$

Note that we consider just connected graphs formed by a finite number of compact intervals. The asymptotic behaviour of the spectrum determines the Euler characteristics of the underlying metric graph $[1,2]$.

The lowest eigenvalue for the standard Laplacian is $\lambda_{1}=0$ is simple provided the graph is connected. The corresponding eigenfunction is $\psi_{1} \equiv 1$. The spectral gap — the difference between the two lowest eigenvalues $\lambda_{2}-$ 
$\lambda_{1}$ - coincides with $\lambda_{2}$. Our interest in investigating the spectral gap in connection to the structure of the metric graph is motivated by its discrete counterpart, where it was called algebraic connectivity by Fiedler [16].

The spectral gap for quantum graphs has been studied intensively during recent years. The first observation was that $[10,17,18]$ :

$$
\lambda_{2}(\Gamma) \geq\left(\frac{\pi}{\mathcal{L}}\right)^{2} .
$$

This result was extended in $[4,10]$ for two-connected (or equivalently bridgeless) graphs

$$
\lambda_{2} \geq 4\left(\frac{\pi}{\mathcal{L}}\right)^{2} .
$$

The difference between these two estimates is usually explained by the fact that the circle is "more connected" than the interval. One may carry out the following experiment. Let us consider what happens to the spectral gap if two intervals of lengths $a$ and $b$ are glued together. The obtained graph is a circle of length $a+b$ (Fig. 1) and the following inequality holds:

$$
\lambda_{2}(\Gamma)=\left(\frac{2 \pi}{a+b}\right)^{2} \geq \min \left\{\lambda_{2}([0, a]), \lambda_{2}([0, b])\right\} .
$$

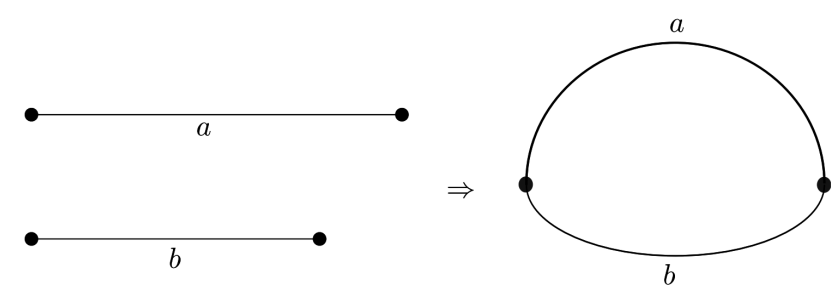

Fig. 1. Gluing together two segments of lengths $a$ and $b$.

This behaviour is surprising, since the spectral gap has a tendency to decrease as the length of the graph increases (due to Weyl's asymptotics). Hence when two graphs are glued together one expects that the spectral gap decreases as in the case where the intervals are joined together at one point

$$
\lambda_{2}([0, a+b])=\left(\frac{\pi}{a+b}\right)^{2} \leq \min \left\{\lambda_{2}([0, a]), \lambda_{2}([0, b])\right\} .
$$

Our goal is to understand this phenomenon in more detail by investigating behaviour of the spectral gap when two graphs are glued together. Our approach is based on the corresponding Titchmarsh-Weyl $M$-function and the answer is given in terms of this function.

An alternative approach was developed in [5, 8, 9, 11], where spectral properties of graphs in relation to their connectivity are studied.

\section{Titchmarsh-Weyl $M$-function: definitions and explicit formulae}

The classical Titchmarsh-Weyl function $M(\lambda)$ was introduced for the one-dimensional Schrödinger operator on the semi-axis $[0, \infty)$ by the following formula ([19], see also [20] for the history and further references):

$$
M(\lambda)=u^{\prime}(k, 0) / u(k, 0),
$$

where $u(k, x)$ is any nontrivial square integrable solution of the corresponding stationary Schrödinger differential equation

$$
-u_{x x}^{\prime \prime}(k, x)+q(x) u(k, x)=\lambda u(k, x), \quad k^{2}=\lambda .
$$

The semi-axis $[0, \infty)$ can be considered as a quantum graph with the natural boundary point $x=0$.

In order to generalise this definition for an arbitrary quantum graph one should first choose a certain vertex set $\partial \Gamma \subset \boldsymbol{V}$ - to be called the boundary of $\Gamma$. Let us denote by $B$ the number of points in $\partial \Gamma$. All vertices in $\Gamma$ that do not belong to $\partial \Gamma$ will be called internal vertices. Consider now any function $u$, which is a solution to the differential Eq. (3.2) on every edge, continuous on $\Gamma$ and satisfies the balance Eq. (2.1) at all internal vertices, i.e. vertices from $\boldsymbol{V} \backslash \partial \Gamma$. It is very important to realise that every such function $u$ is assumed to be continuous not only at internal vertices $\boldsymbol{V} \backslash \partial \Gamma$ but at the boundary vertices $\partial \Gamma$ as well. For $\Im \lambda \neq 0$ such solution is uniquely determined by its values at the boundary points $\left.u\right|_{\partial \Gamma} \in \mathbb{C}^{B}$. Hence it is straightforward to define the matrix valued function

$$
M^{\Gamma}(\lambda):\left.\left.u\right|_{\partial \Gamma} \mapsto \partial u\right|_{\partial \Gamma} .
$$

The relation is linear, therefore $M^{\Gamma}(\lambda)$ will be identified with the corresponding $B \times B$ matrix, where $B$ is the number of points in $\partial \Gamma$. For simplicity, we are going to skip the dependence of $M^{\Gamma}(\lambda)$ upon the choice of the boundary set.

Most important properties of this matrix-valued function are summarised below:

- $M^{\Gamma}(\lambda)$ is a $B \times B$ matrix valued HerglotzNevanlinna function (see definition in [21]) with a discrete set of zeroes and singularities on the positive real axis.

- $M$-function is well-defined for almost all real $\lambda$. For every such $\lambda \in \mathbb{R}, M(\lambda)$ is a Hermitian matrix and therefore it has precisely $B$ real eigenvalues.

Let us remind the following:

Definition 1. A matrix-valued function $\boldsymbol{M}(\lambda)$ is called Herglotz-Nevanlinna if and only if

(1) it is analytic outside the real axis $\lambda \in \mathbb{R}$;

(2) it has positive imaginary part in the upper halfplane

$\Im \boldsymbol{M}(\lambda) \geq 0$, provided $\Im \lambda>0$;

(3) it is symmetric with respect to the real axis

$$
M^{*}(\lambda)=\boldsymbol{M}(\bar{\lambda}) \text {. }
$$

Using general theory of finite rank singular perturbations [22] one may prove the following explicit formula for the Titchmarsh-Weyl function.

Theorem 1. Let us denote by $\mu_{n}$ and $\psi_{n}$ the eigenvalues and normalised eigenfunctions of the standard Laplacian on $\Gamma$, then it holds 


$$
\begin{aligned}
& M^{\Gamma}(\lambda)= \\
& -\left(\sum_{n=1}^{\infty} \frac{\left.\left\langle\left.\psi_{n}\right|_{\partial \Gamma}, \cdot\right\rangle_{\mathbb{C}^{B}} \psi_{n}\right|_{\partial \Gamma}}{\mu_{n}-\lambda}\right)^{-1},
\end{aligned}
$$

where $\left.\psi_{n}\right|_{\partial \Gamma} \in \mathbb{C}^{B}$ denotes the restriction of the eigenfunction $\psi_{n}$ to the boundary $\partial \Gamma-$ a finite dimensional vector; and $\langle\cdot, \cdot\rangle_{\mathbb{C}^{B}}$ is the scalar product in $\mathbb{C}^{B}$.

Proof. The proof is straightforward if one takes into account that the resolvent kernel $r_{\lambda}(x, y)$ of the Schrödinger operator on $\Gamma$ can be written as

$$
r_{\lambda}(x, y)=\sum_{n=1}^{\infty} \frac{\overline{\psi_{n}(y)} \psi_{n}(x)}{\mu_{n}-\lambda} .
$$

Then the function $u$ appearing in the definition of the $M$-function (3.2) can be expressed as

$$
u(x)=-\sum_{n=1}^{\infty} \frac{\psi_{n}(x)}{\mu_{n}-\lambda} \sum_{y \in \partial \Gamma} \overline{\psi_{n}(y)} u^{\prime}(y) .
$$

This gives us (3.6).

Note that the vectors $\left.\psi_{n}\right|_{\partial \Gamma}$ do not form any orthonormal basis in $\mathbf{C}^{B}$, but span the whole space up.

All properties of $M$-functions listed above follow directly from this formula, moreover we see that:

(1) The $M$-function is determined by the traces of the standard eigenfunctions on the boundary $\partial \Gamma$, no knowledge of the eigenfunctions "inside" the graph $\Gamma$ is required.

(2) Only those eigenfunctions that are not identically equal to zero on the boundary contribute into the $M$ function.

(3) The $M$-function is invertible for all $\lambda \in \mathbb{C} \backslash \mathbb{R}$ and almost all $\lambda \in \mathbb{R}$.

A similar result can be obtained using the Dirichlet eigenfunctions - the eigenfunctions of the Laplacian on $\Gamma$ defined on the functions satisfying the Dirichlet vertex conditions on the boundary $\partial \Gamma$ and standard conditions at all internal vertices.

Theorem 2. Let us denote by $\mu_{n}^{D}$ and $\psi_{n}^{D}$ the eigenvalues and normalised eigenfunctions of the standard Laplacian on $\Gamma$ with the Dirichlet conditions on $\partial \Gamma$, then it holds

$$
\begin{aligned}
& M^{\Gamma}(\lambda)-M^{\Gamma}\left(\lambda^{\prime}\right)=\sum_{n=1}^{\infty} \frac{\lambda-\lambda^{\prime}}{\left(\mu_{n}^{D}-\lambda\right)\left(\mu_{n}^{D}-\lambda^{\prime}\right)} \\
& \quad \times\left.\left\langle\left.\partial \psi_{n}^{D}\right|_{\partial \Gamma}, \cdot\right\rangle_{\mathbb{C}^{B}} \partial \psi_{n}^{D}\right|_{\partial \Gamma},
\end{aligned}
$$

where $\left.\partial \psi_{n}^{D}\right|_{\text {дг }}$ denote the vectors of normal derivatives of the Dirichlet eigenfunctions on the boundary $\partial \Gamma$ and $\langle\cdot, \cdot\rangle_{\mathbb{C}^{B}}$ is the scalar product in $\mathbb{C}^{B}$.

The proof of this theorem is completely analogous to the proof of Theorem 1, but instead of the standard Schrödinger equation one needs to use the Dirichlet Schrödinger operator [14].

We see that the Dirichlet spectral data allow one to determine $M$-function up to a constant matrix. One may combine the two theorems to get the following explicit formula:

$$
\begin{gathered}
M^{\Gamma}(\lambda)=\underbrace{-\left(\sum_{n=1}^{\infty} \frac{\left.\left\langle\left.\psi_{n}\right|_{\partial \Gamma}, \cdot\right\rangle \psi_{n}\right|_{\partial \Gamma}}{\mu_{n}}\right)^{-1}}_{M^{\Gamma}(0)} \\
+\underbrace{\left.\sum_{n=1}^{\infty} \frac{\lambda}{\mu_{n}^{D}\left(\mu_{n}^{D}-\lambda\right)}\left\langle\left.\partial \psi_{n}^{D}\right|_{\partial \Gamma}, \cdot\right\rangle_{\mathbb{C}^{B}} \partial \psi_{n}^{D}\right|_{\partial \Gamma}}_{M^{\Gamma}(\lambda)-M^{\Gamma}(0)} .
\end{gathered}
$$

One should use this formula with certain care, since $\mu=0$ is an eigenvalue of the standard Laplacian on $\Gamma$. Therefore the matrix $\sum_{n=1}^{\infty} \frac{\left.\left\langle\left.\psi_{n}\right|_{\partial \Gamma}, \cdot\right\rangle \psi_{n}\right|_{\partial \Gamma}}{\mu_{n}}$ is singular, but its inverse is well-defined. One should understand $M^{\Gamma}(0)$ as the following $\operatorname{limit}_{\lambda \rightarrow 0^{-}} M^{\Gamma}(\lambda)$.

We may draw similar conclusions:

(1) The $M$-function is determined by the traces of the Dirichlet eigenfunctions on the boundary $\partial \Gamma$, no knowledge of the eigenfunctions "inside" the graph $\Gamma$ is required.

(2) Only the Dirichlet eigenfunctions having not identically equal to zero normal derivatives on the boundary contribute to the $M$-function.

We would like to stress that the functions $\psi_{n}$ and $\psi_{n}^{D}$ are eigenfunctions of the Schrödinger operators on $\Gamma$ with different vertex conditions on $\partial \Gamma$. Hence these functions are normalised as elements of the Hilbert space $L_{2}(\Gamma)$. Their restrictions to the boundary $\partial \Gamma$, the vectors $\left.\psi_{n}\right|_{\partial \Gamma}$ and $\partial \psi_{n}^{D} \mid \partial \Gamma$, do not form any orthonormal system in $\mathbb{C}^{B}$.

Formulae (3.6) and (3.7) imply that the eigenvalues of the standard Laplacian on $\Gamma$ can be seen in some sense as zeroes of the $M$-function, while the eigenvalues of the Dirichlet Laplacian correspond to the singularities of $M$. One has to be careful with the definitions, since we are dealing with matrix-valued functions. In particular, zeroes and singularities may occur simultaneously.

\section{4. $M$-function and the spectrum}

We are going to use the following natural definitions: Definition 1. The Titchmarsh-Weyl function $M(\lambda)$ has a singularity at $\lambda=\mu$ iff there exists $\boldsymbol{b}$ such that

$$
\|M(\lambda) \boldsymbol{b}\| \rightarrow \infty \text { as } \lambda \rightarrow \mu
$$

holds. The multiplicity of the singularity is equal to the dimension of the subspace spanned by all vectors $\boldsymbol{b}$ satisfying (4.1).

Definition 2. Titchmarsh-Weyl function $M(\lambda)$ has a generalised zero at $\mu$ iff $-M^{-1}(\lambda)$ has a singularity at $\mu$. The multiplicity of the zero coincides with the multiplicity of the corresponding singularity.

Using these definitions we may provide explicit characterisation of some part of the spectrum using $M$ functions. Typical behaviour of the energy curves for $M^{\Gamma}(\lambda)$ can be seen in Figs. 2 and 3 in the cases $B=2$ and $B=3$.

Theorem 3. The Titchmarsh-Weyl $M$-function determines certain eigenvalues of the standard and Dirichlet 
Laplacians as follows:

1. If $\lambda_{0}$ is a generalised zero of $M^{\Gamma}$, then $\lambda_{0}$ belongs to the spectrum of the standard Laplacian;

2. If $\lambda_{0}$ is a singular point for $M^{\Gamma}$, then $\lambda_{0}$ belongs to the spectrum of the standard Dirichlet Laplacian;

3. The interval $(-\infty, 0)$ is free from zeroes and singularities;

4. The point $\lambda=0$ is a generalised zero and therefore an eigenvalue of the standard Laplacian;

5. The matrix-valued function $M^{\Gamma}(\lambda)$ is negative on $(-\infty, 0)$, i.e. it satisfies the inequality $M^{\Gamma}(0) \leq 0$;

6. Between the singularities, the eigenvalue curves of $M^{\Gamma}(\lambda)$ are monotone and analytic functions of $\lambda$.

Proof. Most of the statements are direct corollaries of the formulae (3.6) and (3.7). One needs to take into account that the standard Laplacian is non-negative with the unique eigenfunction $\psi_{1}(x) \equiv 1$ corresponding to the lowest eigenvalue $\lambda_{1}=0$ (since we assume that the Graph $\Gamma$ is connected). This eigenfunction has trace $\left.\psi_{1}\right|_{\partial \Gamma}=$ $(1,1, \ldots, 1)$ and can be seen from $M^{\Gamma}$. The standard Dirichlet Laplacian is a positive operator.

Monotonicity of the eigenvalue curves follow from the following formula, that is obtained by differentiating (3.8):

$$
\frac{\partial}{\partial \lambda} M^{\Gamma}(\lambda)=\left.\sum_{n=1}^{\infty} \frac{1}{\left(\mu_{n}^{D}-\lambda\right)^{2}}\left\langle\left.\partial \psi_{n}^{D}\right|_{\partial \Gamma}, \cdot\right\rangle_{\mathbb{C}^{B}} \partial \psi_{n}^{D}\right|_{\partial \Gamma}
$$

implying that $\frac{\partial}{\partial \lambda} M^{\Gamma}(\lambda)$ is a sum of projectors with positive coefficients.

Note that this theorem does not imply that all eigenvalues of the standard and standard Dirichlet Laplacians can be determined from the Titchmarsh-Weyl function. In what follows we are going to prove that the lowest eigenvalues can be seen from $M^{\Gamma}$. We first prove the following crucial lemma showing how one may determine the zeroes and singularities.

It will be convenient to denote by $\sigma\left(M^{\Gamma}(\lambda)\right)$ the spectrum of the Hermitian matrix $M^{\Gamma}(\lambda)$ for real nonsingular $\lambda$.

Lemma 1. (Lemma 4.7 from [12]) The number $r(x)$ of generalised zeroes (counted with multiplicities) of $M^{\Gamma}$ to the left of any point $\lambda_{0} \in \mathbb{R}$ can be calculated using the following formula

$$
\begin{aligned}
& r\left(\lambda_{0}\right)=\sum_{\mu_{n}^{D} \leq \lambda_{0}} \tilde{m}^{D}\left(\mu_{n}^{D}\right) \\
& \quad+\lim _{\epsilon \rightarrow 0} \#\left\{\text { positive eigenvalues of } M^{-1}\left(\lambda_{0}-\epsilon\right)\right\},
\end{aligned}
$$

where $\tilde{m}^{D}\left(\mu_{n}^{D}\right)$ is the dimension of the detectable Dirichlet eigensubspace (i.e. the dimension of the subspace in $\mathbb{C}^{B}$ spanned by $\left.\left.\partial \psi(\lambda)\right|_{\partial \Gamma}\right)$ corresponding to $\mu_{n}^{D}$.
Proof. Let us use formula (3.6) to see that $M^{\Gamma}$ has the following structure near the singularities:

$$
M^{\Gamma}(\lambda)=\frac{1}{\mu_{n}^{D}-\lambda} C_{n}+\underbrace{F(\lambda)}_{\text {analytic }},
$$

where $C_{n}$ is a nonnegative Hermitian matrix, its rank is equal to the dimension $\tilde{m}^{D}\left(\mu_{n}^{D}\right)$ of the detectable Dirichlet eigensubspace. For every $\lambda$ between the singularities of $M^{\Gamma}$ there are precisely $B$ eigenvalues $x_{j}(\lambda)$ of $M^{\Gamma}(\lambda)$ :

$$
x_{j}(\lambda) \in \sigma\left(M^{\Gamma}(\lambda)\right) .
$$

In the region $\lambda<0$ we define $y_{j}(\lambda)=\arctan x_{j}(\lambda)$. Their values are in the interval $(-\pi / 2,0)$. For general $\lambda$ each curve $y_{j}(\lambda)=\arctan x_{j}(\lambda)$ is defined as continuous and monotone. One has to be careful just at the singularities of $M^{\Gamma}$, between the singularities the corresponding energy curves are naturally monotone and continuous. In this way we get precisely $B$ monotone curves in the half-plane $(\lambda, y) \in(-\infty, \infty) \times[-\pi / 2, \infty)$.

Zeroes of $M^{\Gamma}(\lambda)$ correspond to those $\lambda$ for which one of the modified curves crosses one of the lines $y=\pi n, n=$ $0,1,2 \ldots$ Hence the number of zeroes to the left of any regular $\lambda_{0}$ is equal to the number of positive eigenvalues of $M^{\Gamma}\left(\lambda_{0}\right)$ plus the number of times the modified energy curves cross the lines $y=\pi / 2+\pi n, n=0,1,2 \ldots$ The second sum is equal to $\sum_{\mu_{n}^{D} \leq x} \tilde{m}^{D}\left(\mu_{n}^{D}\right)$. To cover even not regular $\lambda_{0}$ the formula should be modified as (4.3).

This lemma gives us a tool to estimate the lowest eigenvalues for any graph $\Gamma$, since the lowest eigenvalues are always detectable due to a generalisation of the Courant theorem

Theorem 4. (see [23], also included in [12]). The ground state eigenfunction $\psi_{1}$ chosen nonnegative satisfies

- for the standard Laplacian

$$
\left.\psi_{1}(x) \neq 0 \quad \text { (i.e. } \psi_{1}(x)>\delta>0, x \in \Gamma\right) \text {; }
$$

- for the standard Dirichlet Laplacian with Dirichlet conditions introduced on a subset of vertices denoted by $\partial \Gamma$

$\psi_{1}^{D}(x)=0 \Leftrightarrow x \in \partial \Gamma$,

provided the graph $\Gamma \backslash \partial \Gamma$ is also connected.

The theorem implies in particular that the traces $\left.\partial \psi_{1}^{D}(\lambda)\right|_{\partial \Gamma}$ are different from zero, since otherwise the eigenfunction is equal to zero on the edges directly connected to the boundary points. Hence the lowest Dirichlet eigenvalues always contribute to formula (3.7).

\section{Gluing procedure and the spectral gap}

Consider two metric graphs $\Gamma_{1}$ and $\Gamma_{2}$. Pick up two sets of vertices $\partial \Gamma_{j}=\left\{V_{m}\left(\Gamma_{j}\right)\right\}_{m=1}^{B}$ - of the same size. Then the glued graph $\Gamma$ is the union of the original graphs $\Gamma_{1} \cup \Gamma_{2}$ with points belonging to $\partial \Gamma_{1}$ and $\partial \Gamma_{2}$ identified pairwise. We are going to use the following notation:

$\Gamma=\Gamma_{1} \sqcup_{\partial} \Gamma_{2}$, 
assuming that the boundaries of the original graphs and the way they are glued together are clear.

We are interested in the behaviour of the spectral gap under the gluing procedure. As we know, the eigenvalues of a quantum graph are inverse proportional to the squared total length. Hence it is natural to expect that the spectral gap decreases under gluing since the total length of the glued graph is obviously larger than the lengths of each of the original graphs. It appears that this is not always the case, therefore let us investigate under which conditions the spectral gap becomes larger under gluing.

The answer to this question can be given in terms of the corresponding $M$-functions. Let us first note that $M$-function for the glued graph is just equal to the sum of $M$-functions associated with the original parts:

$$
M^{\Gamma_{1} \sqcup_{\partial} \Gamma_{2}}(\lambda)=M^{\Gamma_{1}}(\lambda)+M^{\Gamma_{2}}(\lambda),
$$

of course provided $\partial \Gamma$ is just the set of glued together vertices. This formula holds only because we assume standard vertex conditions at the vertices of the glued graph.

It follows that all singularities remain and their multiplicities are just multiplicities of the detectable eigenfunctions of the parts. Therefore one may easily calculate the first sum in (4.3). Generalised zeroes on opposite are not preserved. A generalised zero $\lambda_{0}$ is preserved if and only if the traces of the two eigenfunctions on $\Gamma_{1}$ and $\Gamma_{2}$ are parallel

$$
\left.\psi^{\Gamma_{1}}\left(\lambda_{0}\right)\right|_{\partial \Gamma_{1}}=\left.\psi^{\Gamma_{2}}\left(\lambda_{0}\right)\right|_{\partial \Gamma_{2}} .
$$

The following theorem can be proven:

Theorem 5. (Theorem 5.2 from [12]). The spectral gap of the standard Laplacian increases under the gluing procedure if and only if one of the following two conditions is satisfied:

$$
\lambda_{2}(\Gamma)>\min _{j}\left\{\lambda_{2}\left(\Gamma_{j}\right)\right\},
$$

if one of the following two conditions is satisfied:

(1) $\min _{j}\left\{\lambda_{2}\left(\Gamma_{j}\right)\right\}<\min _{j}\left\{\lambda_{1}^{D}\left(\Gamma_{j}\right)\right\}$ and $M\left(\min _{j}\left\{\lambda_{2}\left(\Gamma_{j}\right)\right\}\right.$ has exactly one positive eigenvalue;

(2) $\min _{j}\left\{\lambda_{1}^{D}\left(\Gamma_{j}\right)\right\} \leq \min _{j}\left\{\lambda_{2}\left(\Gamma_{j}\right)\right\}<\max _{j}\left\{\lambda_{1}^{D}\left(\Gamma_{j}\right)\right\}$ and $\lim _{\epsilon \searrow 0} \#\left\{\right.$ positive eigenvalues of $\left.M\left(\min _{j}\left\{\lambda_{2}\left(\Gamma_{j}\right)\right\}+\epsilon\right)\right\}$ $=0$. Of course, the role of graphs $\Gamma_{1}$ and $\Gamma_{2}$ can be exchanged. The spectral never grow if there is just one gluing point. This may be explained by the fact that such gluing does not increase connectivity of the graph. In what follows we illustrate this theorem by providing explicit examples when the spectral gap is growing.

\section{Examples}

\subsection{Gluing two segments}

This is an illustration to the second case in Theorem 5 . Consider two segments of lengths $a=1$ (graph $\Gamma_{1}$ ) and $b=0.5$ (graph $\Gamma_{2}$ ) joined together as shown in Fig. 1.

The corresponding $M$-functions are plotted in Fig. 2 with $k$-variable on the horizontal axis. The lengths are adjusted so that
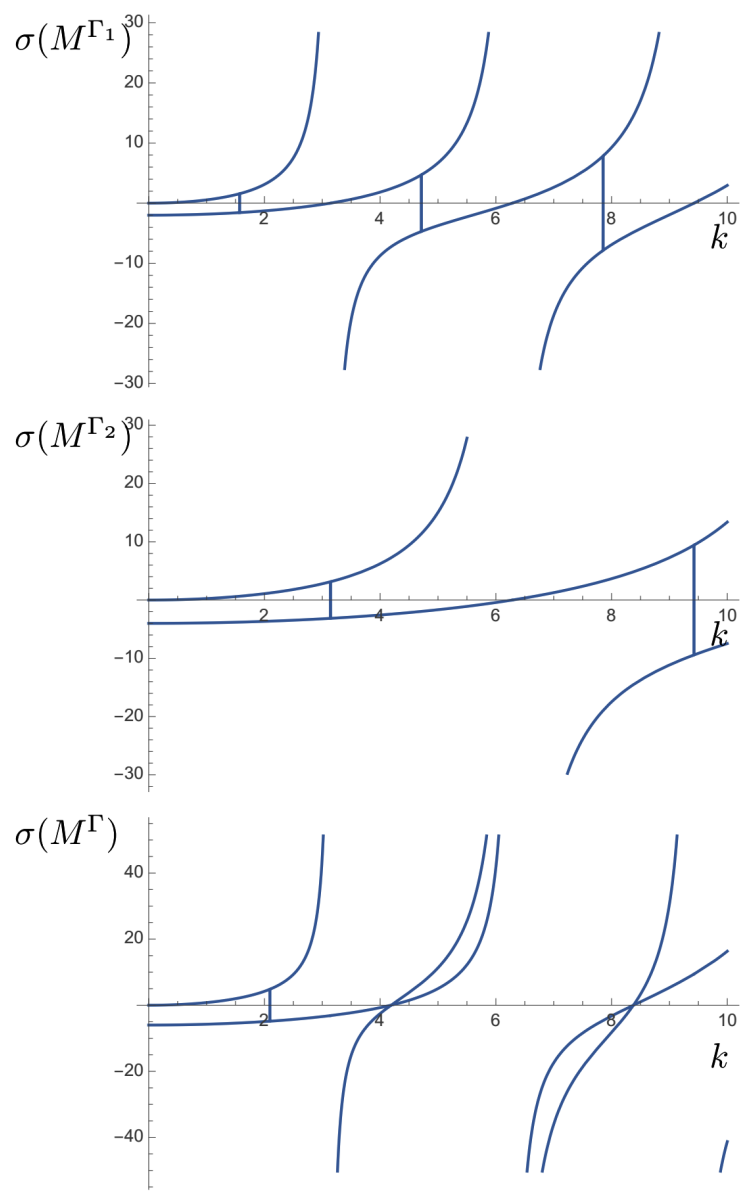

Fig. 2. Eigenvalue curves for two segments of lengths 1 and 0.5 and the loop of length 1.5 .

$$
\lambda_{2}\left(\Gamma_{1}\right)=\lambda_{1}^{D}\left(\Gamma_{1}\right)=\pi^{2}, \lambda_{2}\left(\Gamma_{2}\right)=\lambda_{1}^{D}\left(\Gamma_{2}\right)=(2 \pi)^{2} .
$$

In addition we have that just to the right of the point $\pi=$ $k_{2}\left(\Gamma_{1}\right)=\sqrt{\lambda_{2}\left(\Gamma_{1}\right)}$ the function $M^{\Gamma}$ has two negative eigenvalues. As the result we have

$$
\lambda_{2}(\Gamma)=(3 / 2 \pi)^{2}>\pi^{2}=\lambda_{2}\left(\Gamma_{1}\right) .
$$

It is clear that the cycle graph has higher connectivity than the segments.

\subsection{Gluing two 3-stars}

This is an illustration for the first case in Theorem 5 . Consider two 3-star graphs with edge lengths 2,1.5, 1 and 0.4, 0.2, 0.5 glued together as shown in Fig. 3.

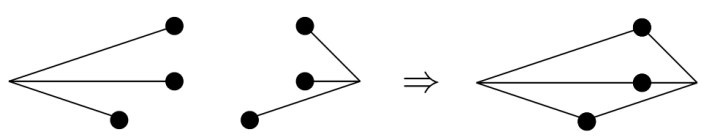

Fig. 3. Gluing together two star graphs with edge lengths $2,1.5,1$ and $0.4,0.2,0.5$ and the glued graph.

The corresponding $M$-functions are plotted in Fig. 4 using variable $k$ again. The lengths of the edges are cho- 


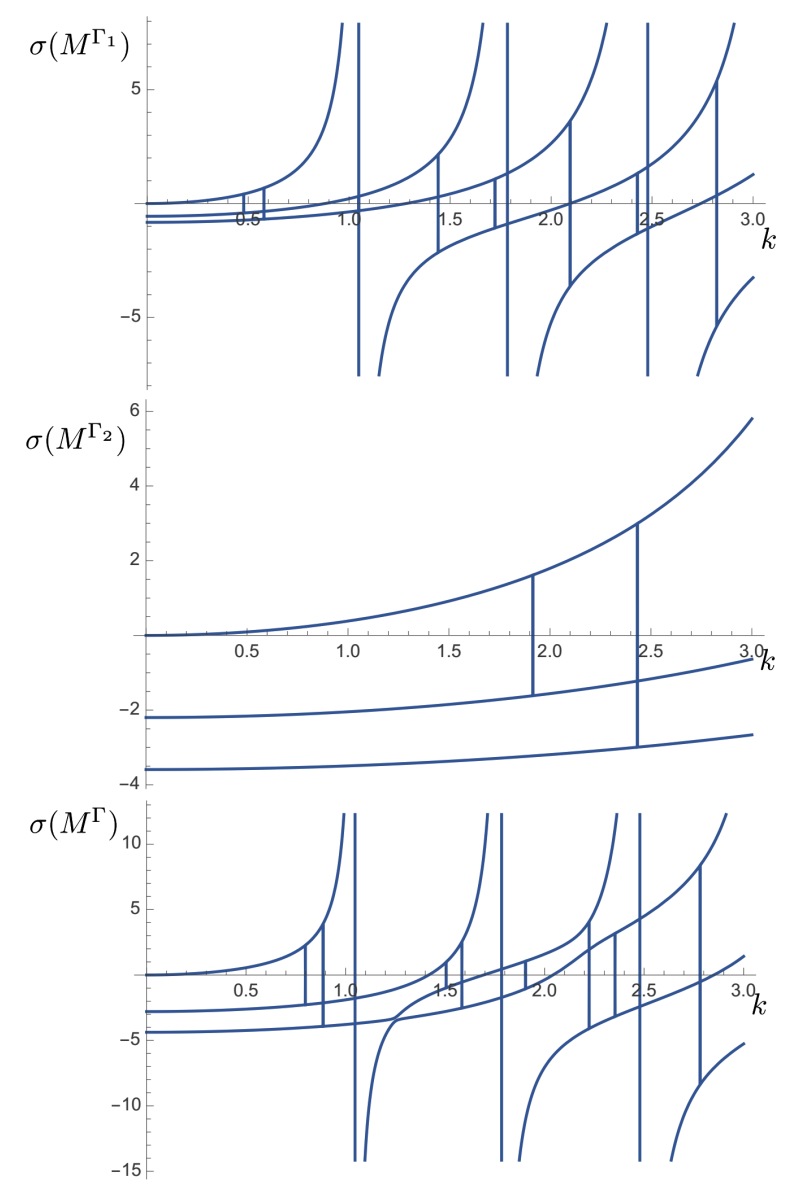

Fig. 4. Eigenvalue curves for star graphs with edge lengths $2,1.5,1$ and $0.4,0.2,0.5$ and for the glued graph.

sen so that $\underbrace{\lambda_{2}\left(\Gamma_{1}\right)}_{(\sim 1.05)^{2}}>\underbrace{\lambda_{1}^{D}\left(\Gamma_{1}\right)}_{(\sim 0.8)^{2}}$. It is easy to see that $M^{\Gamma}\left(\lambda_{2}\left(\Gamma_{1}\right)\right.$ has precisely one positive eigenvalue. It follows that the spectral gap increases under gluing.

\section{Perspectives}

We would like to mention that similar results holds true for the Schrödinger operators and general vertex conditions. The tool we developed can be used to analyse arbitrary graphs if necessary. For example it can be used to estimate the number of eigenvalues similar to [24].

\section{Acknowledgments}

The author thanks the anonymous referee for creative remarks and suggestions leading to improvements of the manuscript.

The author would like to thank Sergey Naboko for stimulating discussions. The work was supported by the Center for Interdisciplinary Research $(\mathrm{ZiF})$ in Bielefeld in the framework of the cooperation group on "Discrete and continuous models in the theory of networks" and by the Swedish Research Council grant D0497301.

\section{References}

[1] P. Kurasov, Ark. Mat. 46, 95 (2008).

[2] P. Kurasov, J. Func. Anal. 254, 934 (2008).

[3] M. Aizenman, H. Schanz, U. Smilansky, S. Warzel, arXiv:1710.07958.

[4] R. Band, G. Lévy, Ann. Henri Poincaré 18, 3269 (2017).

[5] G. Berkolaiko, J. Kennedy, P. Kurasov, D. Mugnolo, J. Phys. A 50, 365201 (2017).

[6] G. Berkolaiko, W. Liu, J. Math. Anal. Appl. 445, 803 (2017).

[7] N.T. Do, P. Kuchment, B. Ong, On resonant spectral gaps in quantum graphs. Functional analysis and operator theory for quantum physics, EMS Ser. Congr. Rep., Eur. Math. Soc., Zürich 2017, p. 213.

[8] J. Kennedy, P. Kurasov, G. Malenova, D. Mugnolo, Ann. Henri Poincaré 17, 2439 (2016).

[9] P. Kurasov, G. Malenová, S. Naboko, J. Phys. A 46, 275309 (2013).

[10] P. Kurasov, S. Naboko, J. Spectral Theory 4, 211 (2014).

[11] J. Rohleder, Proc. Am. Math. Soc. 145, 2119 (2017).

[12] P. Kurasov, S. Naboko, Surgery of graphs and spectral gap: Titchmarsh-Weyl operator-function approach, preprint 2017.

[13] G. Berkolaiko, P. Kuchment, Introduction to Quantum Graphs. Mathematical Surveys and Monographs, Vol. 186, American Mathematical Society, Providence (RI), 2013.

[14] P. Kurasov, Quantum graphs: spectral theory and inverse problems, to appear in Brikhäuser, 2018.

[15] O. Post, Spectral Analysis on Graph-Like Spaces, Lecture Notes in Mathematics, Vol. 2039, Springer, Heidelberg 2012.

[16] M. Fiedler, Czechoslov. Math. J. 23 (98), 298 (1973).

[17] S. Nicaise, Bull. Sci. Math. 111, 401 (1987).

[18] L. Friedlander, Ann. Inst. Fourier 55, 199 (2005).

[19] H. Weyl, Math. Ann. 71, 441 (1912) (in German).

[20] W.N. Everitt, J. Comput. Appl. Math. 171, 185 (2004).

[21] F. Gesztesy, E. Tsekanovskii, Math. Nachr. 218, 61 (2000).

[22] S. Albeverio, P. Kurasov, Singular Perturbations of Differential Operators. Solvable Schrödinger Type Operators, London Mathematical Society Lecture Note Series, Vol. 271, Cambridge University Press, Cambridge 2000 .

[23] R. Band, P. Kurasov, On Courant Theorem for Quantum Graphs, in preparation.

[24] J. Behrndt, A. Luger, J. Phys. A 43, 474006 (2010). 